\title{
Investigating the health care delivery system in Japan and reviewing the local public hospital reform
}

\author{
This article was published in the following Dove Press journal: \\ Risk Management and Healthcare Policy \\ 18 March 2016 \\ Number of times this article has been viewed
}

\section{Xing Zhang \\ Tatsuo Oyama}

National Graduate Institute for Policy Studies, Tokyo, Japan
Correspondence: Tatsuo Oyama;

\section{Xing Zhang}

National Graduate Institute for

Policy Studies, 7-22-I Roppongi,

Minato-ku, Tokyo 106-8677, Japan

Tel +8I 364396000

Fax +8I 364396010

Email oyamat@grips.ac.jp;

tonyxingzhang@gmail.com

\begin{abstract}
Japan's health care system is considered one of the best health care systems in the world. Hospitals are one of the most important health care resources in Japan. As such, we investigate Japanese hospitals from various viewpoints, including their roles, ownership, regional distribution, and characteristics with respect to the number of beds, staff, doctors, and financial performance. Applying a multivariate analysis and regression model techniques, we show the functional differences between urban populated prefectures and remote ones; the equality gap among all prefectures with respect to the distribution of the number of beds, staff, and doctors; and managerial differences between private and public hospitals. We also review and evaluate the local public hospital reform executed in 2007 from various financial aspects related to the expenditure and revenue structure by comparing public and private hospitals. We show that the 2007 reform contributed to improving the financial situation of local public hospitals. Strategic differences between public and private hospitals with respect to their management and strategy to improve their financial situation are also quantitatively analyzed in detail. Finally, the remaining problems and the future strategy to further improve the Japanese health care system are described.
\end{abstract}

Keywords: health care system, health care resource, public hospital, multivariate regression model, financial performance

\section{Introduction}

Japan's health care system is considered one of the best health care systems in the world for various reasons, including its availability, effectiveness, and efficiency. Over the past 50 years, Japan has achieved satisfactory demographic health at reasonably low cost. ${ }^{1-4}$ The Japanese population has the longest life expectancy among all OECD countries, while health care expenditure, as a share of gross domestic product (GDP), is below most of the developed countries in the OECD. Many researchers attribute the success of the Japanese health care system to policy-makers who have been proficient at balancing demands and supplies with dexterous skill, controlling medical prices under the universal health insurance system. ${ }^{2,5,6}$

Japan established a universal health insurance system in 1961. A goal was set for the health care system, by the Japanese government, to provide equitable access to "necessary and adequate" medical services to the entire population at a relatively low cost. ${ }^{1,3}$ To realize the goal, two key issues have been paid substantial attention. First, the capability of the population to pursue necessary and adequate health services is related to how health care services are financed. The well-established health insurance system has ensured medical services are affordable to every citizen, though it faces 
increasing financial challenges. Second, accessibility to health care services is related to the equal access to health care services, however, is not well solved yet and faces increasing challenges. In this paper, we aimed to investigate the major characteristics of the Japanese health care delivery system, focusing on the local public hospital (LPH) reform process.

The basic health policy of Japan is characterized as a combination of tight control of the payment system and a laissez-faire approach regarding how services are delivered. For the payment system, the supply-side cost control is imposed by a uniform fee schedule at national level; thus, all providers, no matter whether private or public, share the same prices for their medicines, devices, and services under this nationwide fee schedule.

For the health care delivery system, a laissez-faire approach is adopted by the administration. The health care market is open to all domestic participants equally. According to Medical Service Law, ${ }^{7}$ establishing a hospital, defined as a facility with 20 or more beds, requires the approval of the government, while starting a clinic with less than 20 beds simply requires the owner to notify relevant local authorities. The policy has led to two consequences: first, the private sector dominated the health care delivery system as seen by the fact that approximately $80 \%$ of all hospitals and $70 \%$ of all hospital beds were operated by the private sector in $2011 ; 8$ and, second, there were a large number of redundant hospital beds in Japan. Namely, in 2011, the number of hospital beds in general was more than twice the Organization for Economic Co-operation and Development average, despite a significant drop since $1996 .{ }^{9}$

Figure 1 shows the number of hospitals by ownership, such as individual, corporate, social security, local public, national public, and others, from 2002 to 2012. Regarding

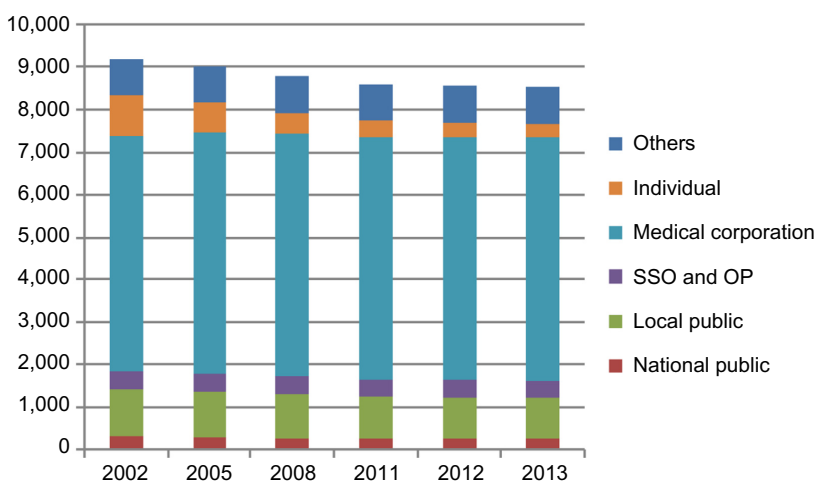

Figure I Number of hospitals by ownership.

Note: Data from Survey of Medical Institutions. Ministry of Health, Labour and Welfare; 2002-20I3. Available from: http://www.mhlw.go.jp/toukei/list/79-Ia.html. Accessed April 15, 2015.8

Abbreviation: SSO, social security organizations; OP, other public hospitals. the total number of hospitals in Japan, we can see a slight decrease over the last 10 years from 9,187 in 2002 to 8,565 in 2012 , a $6.8 \%$ decrease over 10 years. All types of hospitals have shown decreases except for the largest group, corporate/private hospitals, which have shown an increase from 5,533 in 2002 to 5,709 in 2012, a 3.2\% increase over 10 years. From Figure 1, we can see that individual hospitals especially, showed the largest decrease, from 954 in 2002 to 320 in 2012, a decrease of more than $65 \%$.

The health care delivery system in Japan consists of four types of facilities: hospitals, clinics, health centers providing public health services, and pharmacies providing prescription drugs and over-the-counter drugs. In this paper, we mainly focus on hospitals, as they are the main providers of medical services in Japan. The Government of Japan adopts a neutral position in the administration of the health care market. The competition among hospitals has been intense in recent years as the deteriorating national financial situation has further enhanced the efficiency of the private sector-oriented health care delivery system. In order to maintain the equality of the health care delivery system, LPHs are considered one of the most important countermeasures to secure medical services for local residents. The LPHs are owned by local governments and Local Independent Administrative Corporations (LIACs), and aim to promote the welfare of local residents. The LPHs focus more on implementing the so-called "policy-based medical services", which include providing high-tech care for cancer, circulatory and complicated diseases, providing medical care for non-profitable diseases, serving remote areas, training medical staff, and providing disaster relief and other emergency services.

During the first decade of the 21 st century, increased health care demands caused by the aging population and the deteriorating national financial situation led public hospital policy to be at the center of controversy among policy-makers at both the local and national level, as well as among the general public. As one of the largest industries owned by local governments, LPHs' unfavorable financial performance worsened local fiscal situations, which suffered from expanding outlays and huge debt. ${ }^{10}$ Thus, LPH reform was conducted by the Ministry of Internal Affairs and Communications (MIC) in late 2007. Comprehensive measures were included in the reform guidelines ${ }^{10}$ aimed at not only enhancing the fiscal soundness, but also improving the efficiency and accountability of LPHs. Local governments were required to develop a 5-year plan according to the guideline. The results of the LPH reform surveillance data show that the percentage of LPHs operating with a surplus increased from $25.5 \%$ in 2006 
to $51.9 \%$ in 2011, and annual deficits sharply decreased from more than 190 billion yen to minus 5.6 billion yen (surplus) during the same period. In this paper, we investigate the impact of LPHs and their reform quantitatively in more detail, from multiple dimensions, including health care resource allocation, health expenditure, financial performance, efficiency of LPHs, and so on.

In "The health care delivery system in Japan" section, we describe the health care delivery system in Japan focusing upon various types of hospitals. We apply a multivariate analysis and regression model techniques to investigate their regional distribution and the equality issue. In the "LPH reform and its effects" section, we review the LPH reform, then we evaluate its effects from the viewpoint of the expenditure and revenue structure.

\section{The health care delivery system in Japan \\ Regional distribution of hospitals}

Hospitals are the major and most important health care resource in Japan. We classify hospitals into three groups depending upon their ownership: LPHs, other public hospitals (OPHs), and private hospitals (PRHs). LPHs are those operated by prefectures, municipalities, and LIACs; OPHs include national public hospitals and social insurance organizationowned hospitals; and PRHs are those operated by medical corporations, individuals, and others. Table 1 shows the number of hospitals, beds, and staff for each type of hospital in Japan in 2011. PRHs dominate all three categories, for the percentage of hospitals, beds, and staff at $80.8 \%, 69.6 \%$, and $64.5 \%$, respectively. For LPHs, we see the share for the number of hospitals, beds, staff ranging between $11 \%$ and $17 \%$, while for OPHs, the share of the number of hospitals is $4.6 \%$ and for staff is $19.0 \%$. This implies that national hospitals employ more staff per facility compared with the

Table I Number of hospitals, beds, and staff in Japan (201 I)

\begin{tabular}{llll}
\hline Ownership & Hospitals & Beds & Staff \\
\hline LPH, n (\%) & $968(I 1.2)$ & $232,934(14.7)$ & $313,747(16.4)$ \\
Per hospital (n) & & 262.2 & 324.1 \\
OPH, n (\%) & $685(8.0)$ & $248,717(15.7)$ & $362,531(19.0)$ \\
Per hospital (n) & & 363.1 & 529.2 \\
PRH, n (\%) & $6,952(80.8)$ & $1,101,422(69.6)$ & $1,233,460(64.6)$ \\
$\quad$ Per hospital (n) & & 158.4 & 177.4 \\
Total, N (\%) & $8,605(100.0)$ & $1,583,073(100.0)$ & $1,958,018(100.0)$ \\
\hline
\end{tabular}

Note: Data from Survey of Medical Institutions. Ministry of Health, Labour and Welfare; 2002-20I3. Available from: http://www.mhlw.go.jp/toukei/list/79-la.html. Accessed April 15, 2015.8

Abbreviations: $\mathrm{LPH}$, local public hospital; OPH, other public hospital; $\mathrm{PRH}$, private hospital. other types of hospitals. Comparing the range of the three shares for hospitals, beds, and staff, we find that the hospital shares' range is the largest from approximately $8.0 \%$ to $80.8 \%$, with the beds' shares ranging from $14.7 \%$ to $69.6 \%$, and the staff's shares ranging from $16.4 \%$ to $64.6 \%$.

The figures in the lower row in the second and the third columns of Table 1 give the number of beds and staff per corresponding hospital for LPHs, OPHs, and PRHs. The OPHs have the largest scale in terms of both the number of beds and the number of staff per hospital at 363.1 and 529.2, respectively. On the other hand, PRHs show the smallest scale in terms of both the number of beds and the number of staff per hospital compared with LPHs and OPHs. The LPH data remain in between those of OPHs and PRHs, but are much closer to the PRH data rather than the OPH data. Thus, we find that OPHs, eg, national public hospitals and social security-owned hospital with large-scale facilities, focus more on advanced care and comprehensive medical services, while PRHs provide more primary care and basic medical services. The functions of LPHs are between these two types.

We find that among LPHs, OPHs, and PRHs, the order between the largest and smallest shares among hospitals, beds, and staff changes. Namely, LPHs and OPHs have the largest share in staff (16.4\% and 19.0\%, respectively), then bed shares ( $14.7 \%$ and $15.7 \%$, respectively), and then hospital shares $(11.2 \%$ and $8.0 \%$, respectively) are the smallest. On the other hand, PRHs have the largest share in hospitals $(80.8 \%)$, then in beds $(69.6 \%)$, and the staff's share is the smallest (64.6\%). This fact implies that PRHs are rather widely distributed with a relatively smaller number of beds and staff, while public hospitals, LPHs, and OPHs are more concentrated with a relatively large scale with more beds and more staff to provide a wide range of advanced medical services. Thus, we can say that PRHs and LPHOPHs play complementary roles; namely, they are mutually complementary in providing medical services.

Investigating the number of hospitals, beds, staff, and doctors for each prefecture in Japan in 2006 and 2011, respectively, for hospitals and beds, most prefectures have smaller values in 2011 than in 2006. However, for the number of staff and doctors, we find that in most prefectures, the values in 2011 are larger than those in 2006. This indicates that most prefectures addressed the staffing problem without increasing the number of hospitals and beds. We also find that the prefectures having a much larger number of hospitals, beds, and staff are Hokkaido (HKD), Tokyo (TKY), Osaka (OSK), Fukuoka (FKO), Saitama (SIT), Chiba (CHB), Kanagawa (KNG), Aichi (AIC), and Hyogo (HYG), ie, the most urbanized and 
populated prefectures in Japan with the major corresponding large cities of Sapporo, Tokyo, Osaka, Fukuoka, Saitama, Chiba, Yokohama, Nagoya, and Kobe, respectively, in metropolitan and designated populated urban areas. The less developed prefectures, such as Tottori (TTR), Shimane (SMN), and Fukui (FKI), have fewer hospital resources.

We investigate the relationship among the number of hospitals, beds, staff, and doctors by applying regression model analysis techniques. The regression results for each type of function form between two factors are shown in Table 2 . These three factors hospitals, beds, and staff are mutually highly correlated. Regarding the relationship between beds and hospitals for all prefectures for the years 2006 and 2011 in Japan, we find that they are proportionally related to each other. Their proportional coefficients corresponding to the actual average values for the number of beds per hospitals are not significantly different between the years 2006 and 2011 (181.9 and 184.0, respectively). Thus, for the relationship between beds and hospitals, combining the data for 2006 and 2011 and applying the single variable linear function passing the origin, we obtain the estimate for the slope of the linear regression model as 185.5, as shown in Table 2 .

On the relationship between staff and beds, we find that there is a significant difference between the years 2006 and 2011, so the estimate for the slope is given as 1.042 and 1.234 as in Table 2, an almost $20 \%$ increase during the 5 -year period. The actual average number of staff per beds is 1.049 and 1.206 in 2006 and 2011, respectively. Regarding the relationship between doctors and beds, we find that those nine populated prefectures mentioned earlier are remarkably higher than the others with respect to their numbers, while the remaining 38 prefectures are densely distributed in the area with lower numbers. In addition, we find that doctors increases higher than proportional to beds's increase; namely, their relationship can be expressed by a convex (quadratic) function, as shown in Table 2. These relationships indicate that compared to 2006 , the number of hospitals and hospital beds decreased while the number of hospital staff increased in 2011.

The health care resources in Japan are concentrated in these rather "profitable areas", and not in remote areas. Considering that the location of these health care resources is generally and largely determined by the population size, we investigate the relationship between the distribution of health resources and the population density for all prefectures. To measure the equality of health care resources objectively, we take the density of the health care resources into account. From the relationship between hospital density (DHS) and bed density (DBD), given by indices such as the number of hospitals per 100 thousand population and the number of beds per 1,000 population for all prefectures, we find that DHS is much higher in those underdeveloped prefectures located in the southern part of Japan such as KOC, KGS, and TKS, while those populated prefectures like KNG, SIT, and CHB had a lower density of hospitals, hospital beds, and staff. Compared to 2006, we find that the density of hospital staff significantly increased in 2011.

We find that the concave function with parameter estimates 0.629 and 0.639, which are less than 1.0, for 2006 and 2011, respectively, can approximate the actual data better for the relationship between DBD and DHS. This parameter implies that the percentage increase of DBD corresponding to a unit percentage increase of DHS, interpreted as the DBD elasticity with respect to DHS, becomes less as the latter factor, DHS density, increases. Also, we find that the relationship between DST and DBD is linear with a slope larger than 1.0 for both years 2006 and 2011, in which that for 2011 is almost 15\% larger than the 2006 estimate. This implies that we need much more staff per bed recently even with respect to the density per capita. Thus, the same trend is found in hospital resources measured by density; namely, hospitals per 1,000 population tend to have more beds, and beds per 1,000 population tend to have more staff in 2011 compared with 2006 . The density

Table 2 Regression results

\begin{tabular}{|c|c|c|c|c|c|}
\hline Dependent variable & Beds (n) & Staff $(n)$ & Doctors (n) & $\begin{array}{l}\text { DBD (n per I,000 } \\
\text { population) }\end{array}$ & $\begin{array}{l}\text { DST (n per I,000 } \\
\text { population) }\end{array}$ \\
\hline \multirow[t]{2}{*}{ Independent variable } & hospitals (n) & beds (n) & beds (n) & DHS (n per 1,000 & DBD (n per I,000 \\
\hline & & & & population) & population) \\
\hline Model & $y=a x$ & $y=a x$ & $y=a x^{2}+b x$ & $y=a x^{b}$ & $y=a x$ \\
\hline \multicolumn{6}{|l|}{ Parameter estimate } \\
\hline \multirow[t]{2}{*}{$a$} & $185.5 * * *$ & I.042*** (2006) & $0.00052 * * *(2006)$ & 4.24 I*** (2006) & I.025*** (2006) \\
\hline & & $1.234 * * *(201 \mathrm{I})$ & $0.00065 * * *(2011)$ & $4.270 * * *(2011)$ & I.170*** (2011) \\
\hline \multirow[t]{2}{*}{$b$} & - & - & $0.0794 * * *(2006)$ & $0.629 * * *(2006)$ & - \\
\hline & & & $0.0870 * * *(201 \mathrm{I})$ & $0.639 * * *(201 \mathrm{I})$ & \\
\hline \multirow[t]{2}{*}{$\mathrm{R}^{2}$} & 0.9875 & $0.9982(2006)$ & $0.958(2006)$ & $0.878(2006)$ & $0.998(2006)$ \\
\hline & & $0.9952(2011)$ & $0.957(2011)$ & $0.897(2011)$ & $0.997(2011)$ \\
\hline
\end{tabular}

Note: $* * * P<0.01$. - indicates data not available.

Abbreviations: DBD, density of beds; DHS, density of hospitals; DST, density of staff. 
models indicate that the scale of hospitals is relatively small in prefectures with high hospital resource density because the hospital elasticity of beds is only approximately 0.63 , and the coefficients are smaller than those of the non-density models. Prefectures with high hospital resource density are usually the less developed ones, with more geographic barriers and lower population density, which prevent local governments from providing medical services with just a few large hospitals. The results reflect the impacts of the health policy during this period: the number of hospitals and beds decreased, and the beds were concentrated in large hospitals. The average density of hospital staff increased among prefectures because of the policy promoting the number of medical staff, but a substantial number of the increased medical staff was absorbed by more developed prefectures like TKY, OSK, and KNG.

We apply a cluster analysis technique to classify all 47 prefectures into several groups based upon three kinds of data: the number of hospitals, beds, and doctors. Based on the hierarchical cluster analysis technique, which aims at integrating closely located data into groups at each stage, 47 prefectures were clustered into four groups (Table 3 ). Detailed data for each cluster are provided with basic statistical data in Table 4. From Table 4, we find that the prefectures belonging to each cluster in I, II, III, and IV have significantly different mean values. Namely, the average values for all three indices, ie, number of hospitals, beds, and staff, get smaller from cluster I to IV. This means that the four clusters from I to IV are ordered from the largest to the smallest with respect to all these indices. Incidentally, the same clustering is obtained for all cases of separate data for 2006 and 2011, and for the aggregate data for these 2 years.

\section{LPHs distribution and measuring the equity}

Public and private medical facilities receive the same payments according to the uniform fee schedule in Japan.

Table 3 Results of cluster analysis

\begin{tabular}{ll}
\hline Cluster & Prefectures \\
\hline I & HKD, TKY, OSK, FKO \\
II & SIT, CHB, KNG, AIC, HYG \\
III & MYG, FKS, IBR, GNM, NGT, NGN, SZO, KYT, OKY, \\
& HRS, YGC, EHM, KOC, NGS, KMM, OIT, MIZ, KGS \\
IV & AOM, IWT, AKT, YGT, TCG, TYM, ISK, FKI, YMN, GIF, \\
& MIE, SIG, NAR, WKY, TTR, SMN, TKS, KGW, SAG, OKN \\
\hline
\end{tabular}

Abbreviations: HKD, Hokkaido; AOM, Aomori; IWT, Iwate; MYG, Miyagi; AKT, Akita; YGT, Yamagata; FKS, Fukushima; IBR, Ibaraki; TCG, Tochigi; GNM, Gumma; SIT, Saitama; CHB, Chiba; TKY, Tokyo; KNG, Kanagawa; NGT, Niigata; TYM, Toyama; ISK, Ishikawa; FKI, Fukui; YMN, Yamanashi; NGN, Nagano; GIF, Gifu; SZO, Shizuoka; AIC, Aichi; MIE, Mie; SIG, Shiga; KYT, Kyoto; OSK, Osaka; HYG, Hyogo; NAR, Nara; WKY, Wakayama; TTR, Tottori; SMN, Shimane; OKY, Okayama; HRS Hiroshima; YGC, Yamaguchi; TKS, Tokushima; KGW, Kagawa; EHM, Ehime; KOC, Kochi; FKO, Fukuoka; SAG, Saga; NGS, Nagasaki; KMM, Kumamoto; OIT, Oita; MIZ, Miyazaki; KGS, Kagoshima; OKN, Okinawa.
Table 4 Basic statistics for each cluster

\begin{tabular}{|c|c|c|c|c|c|c|}
\hline \multirow[t]{2}{*}{ Cluster } & \multicolumn{2}{|c|}{ Hospitals } & \multicolumn{2}{|l|}{ Beds } & \multicolumn{2}{|l|}{ Doctors } \\
\hline & Mean & SD & Mean & SD & Mean & SD \\
\hline \multirow[t]{2}{*}{ I } & 573.8 & 79.6 & $108,162.3$ & $17,225 . \mid$ & $13,69 \mid .9$ & $6,707.2$ \\
\hline & 555.8 & 74.2 & $105,368.8$ & $17,123.3$ & $15,173.3$ & $7,701.0$ \\
\hline \multirow[t]{2}{*}{ II } & 339.2 & 30.1 & $65,711.8$ & $7,108.7$ & $8,037.3$ & $1,730.0$ \\
\hline & 328.8 & 29.1 & $64,983.8$ & $6,305.0$ & $9,171.9$ & I,957.6 \\
\hline \multirow[t]{2}{*}{ III } & 173.7 & 40.8 & $29,620.3$ & $6,866.2$ & $2,857.9$ & 846.2 \\
\hline & 166.9 & 40.4 & $28,689.7$ & $6,760.2$ & $3,085.3$ & 962.7 \\
\hline \multirow[t]{2}{*}{ IV } & 91.3 & 22.7 & $16,610.8$ & $3,624.1$ & I,739.7 & 428.3 \\
\hline & 86.7 & 21.2 & $16,013.2$ & $3,468.6$ & I,870.6 & 492.2 \\
\hline
\end{tabular}

Abbreviation: SD, standard deviation.

Many public hospitals are compensated for their losses by budget allocations from the national government or local governments because they have to undertake policy-based medical services. National public hospitals focus more on highly advanced medical treatment, clinical research for designing health policies, and the education and training of health workers, while LPHs concentrate more on local needs. The LPHs are owned by local governments, operating in the form of local public enterprises (LPEs), which are established for the purpose of promoting the welfare of local residents. Several fundamental rights, such as budgeting, middle, and long-term planning formulation, and other important administrative powers, are retained by local governments. In 2008, the first 22 LPHs were transferred from local governments to the LIAC. Unlike LPHs operated by local governments, LIACs have a legal status, which allows them to be more independent. ${ }^{11}$

Although the proportion of LPHs is not very large in Japan's health care delivery system, they intensively get involved in performing public functions. According to the data from Ministry of Health Labour and Welfare, at the end of 2011, LPHs only accounted for approximately $11 \%$ of hospitals, $15 \%$ of hospital beds, and $16 \%$ of hospital staff, while they operated approximately $67 \%$ of the infectious disease beds, $23 \%$ of the emergency beds, and $30 \%$ of the tuberculosis beds. The role of LPHs is even substantial within the context of LPEs. Hospitals are one of the most important industries run by LPEs. The LPHs comprise the second largest industry (LPEs are classified as water supply, transportation, and electricity industries, etc, according to their function) in terms of expenditure, which was 4.4 trillion yen, $25 \%$ of total expenditure, and was the largest industry in terms of revenue, which was 3.2 trillion yen, $35.8 \%$ of total revenue.

Regarding the distribution of LPH resources among prefectures in 2011, we find that the number of LPHs is highly correlated with the number of LPH beds. Pearson correlation 
coefficients are 0.861 in 2006 and 0.830 in 2011 . The average number of LPHs in a prefecture is 20.6. The OIT has the smallest number of LPHs, which is only five, while HKD has 98. The average number of LPH beds was 4,956.0, with the lowest number being 1,140.0 in SAG, and the highest number, 15,531.0 in HKD. The average number of LPH staff in a prefecture is 6,675.5. The OIT has the smallest hospital staff, 1,952.2, while HKD has the biggest, $19,006.5$. The average percentage of LPHs in a prefecture is $14.1 \%$; OIT has the lowest percentage of LPHs, while YMG has the highest percentage. They were $3.1 \%$ and $36.8 \%$, respectively. The average percentage of beds owned by LPHs was $17.3 \%$, with the lowest percentage being $5.0 \%$ in $\mathrm{FKO}$, and the highest percentage being 39.0\% in YMG. The average percentage of LPH staff was $19.3 \%$, with the lowest being $5.4 \%$ in FKO and the highest being $43.8 \%$ in YMG.

The results reveal that although the absolute amount of LPH resources in underdeveloped prefectures in the Tohoku area, such as YMG, IWT, and AOM, was lower than that in developed prefectures, such as FKO, OSK, SAG, and KNG, the share of LPH resources in these prefectures was much higher. This reflects the important role of LPHs in these underdeveloped prefectures.

Equity regarding the health care system and the allocation of health care resources has been a great concern for many researchers. A large number of studies have investigated the allocation of human resources in the health care system of Japan. Most of these studies use the Gini index to evaluate whether the health resources are distributed evenly. Many of these research studies apply concentration indices such as the Lorenz curve and the Gini index ${ }^{12,13}$ and the Atkinson and Theil indices. ${ }^{14}$ We consider these approaches using these indices as a "macro approach", while we are focused upon more "micro approaches" in our paper using various kinds of measures in more detail related to hospitals, beds, and staff. Assuming that LPHs have played an important role in maintaining the equity of the health care delivery system in Japan, we believe that the LPH reform launched in late 2007 has had impacts on the equity of this system. Thus, the data after the LPH reform should be analyzed.

Shinjo and Aramaki ${ }^{15}$ used the data in 2008 at the secondary health care delivery service areas (SHSA) level to analyze the factors that influence the distribution of health care resources in Japan in their multiple regression analysis. They found that health care services were significantly scarcer in outflow groups after controlling for demographic and socioeconomic factors. The provision of health care services was also unbalanced among different inpatient flow groups. We believe that collecting cross-sectional data and trying to analyze the effects of time are very important for policy-makers. In this paper, we apply the "gap" analysis technique in order to investigate the distribution of health care resources. We try to measure the scale of the health care resource shortage among prefectures.

We denote the set of health care delivery resources and the set of prefectures by $M=\{$ hospitals, beds, doctors $\}$ and $N=\{1, \ldots, 47(=n)\}$, respectively. Then let $a_{j}$ and $p_{j}$ indicate the area and the population, respectively, for each prefecture $j$, $j \in N=\{1, \ldots, n\}$. We denote the amount of health care delivery resource $i, i \in M$ for each prefecture $j, j \in N$ by $x_{i j}$. Assuming that the health care delivery resource would be allocated proportionally to the area and population, we let $y_{i j}^{A}$ and $y_{i j}^{P}$ be the expected proportional allocation of health resource $i$ in prefecture $j$ based upon the area share and the population share, respectively. Thus, applying the Hamilton method (the largest fraction method) in order to calculate the closest integral value to each of $y_{i j}^{A}$ and $y_{i j}^{P}$, we can write them as follows.

$$
y_{i j}^{A}=\frac{a_{j}}{A} \sum_{j} x_{i j}, \quad y_{i j}^{P}=\frac{p_{j}}{P} \sum_{j} x_{i j}
$$

where $A=\sum_{j} a_{j}$ and $P=\sum_{j} p_{j}$

The Hamilton method first gives an integral value equal to the largest integer not exceeding $y_{i j}^{A}$ and $y_{i j}^{P}$ to each prefecture. Then calculating the descending order data based upon the remainders given by the difference between $y_{i j}^{A}$ or $y_{i j}^{P}$ and the nearest (equal or less than respective value) integer, we give additional resources to all possible prefectures with the largest remainder until the total number of resources is allocated. Denoting the final allocation by the Hamilton method, which we call the proportional allocation, as $\left[y_{i j}^{A}\right]$ and $\left[y_{i j}^{P}\right]$, respectively, we can calculate the gap of the allocation of health care delivery resource $i$ in prefecture $j$ as $x_{i j}^{A}-\left[y_{i j}^{A}\right]$ and $x_{i j}^{P}-\left[y_{i j}^{P}\right]$ , respectively, by taking the difference between the current allocation and the proportional allocation for each resource. From all these gaps corresponding to the health care delivery resources of beds and doctors for each prefecture for 2 years 2006 and 2011, a positive value indicates the prefecture possesses more health care resources than expected by the proportional allocation, while a negative value indicates fewer health care resources than that to be allocated to the corresponding prefecture. We find that the seven most urbanized and populated prefectures in Japan, TKY, OSK, KNG, SIT, FKO, CHB, and AIC, are located far from the other 40 prefectures. Namely, regarding the bed-gap only $\mathrm{FKO}$ has large positive values for both population and area gaps, while TKY, KNG, SIT, CHB, and AIC have positive values for the area gap and a negative 
population gap, and OSK has a positive value for the area gap and no population gap. Especially, both TKY and OSK have large positive values for the area gap. On the other hand, regarding the doctor gap, TKY, OSK, and FKO have large positive values for both the population and area gaps, while KNG, SIT, CHB, and AIC have positive values for the area gap and negative population gaps. In particular, TKY has very large positive values for both the area and population gaps.

Regarding the bed gap among the other 40 prefectures, those in the southern part of Japan, such as KMM, YGC, and NGS, have positive values for both the population and area gaps, while the prefectures with large shares of LPHs, such as IWT, AKT, FKS, and AOM, have positive values for the population gap and negative area gaps. Prefectures, including MYG, YMG, TCG, and GNM have negative values for both the population and area gaps. We also find that the mean of the absolute values of the bed gap decreased from 51,165 in 2006 to 50,357 in 2011 by area and slightly increased from 12,170 to 12,415 by population. Regarding the doctor gap among the other 40 prefectures, as a general trend, we can say that several prefectures shifted from the negative population quadrant to the positive population quadrant, which indicates that more doctors were attracted to those two metropolises. Also, the mean of the absolute value of the doctor gap increased from 6,749 in 2006 to 7,553 in 2011 by area and increased from 1,145 to 1,209 by population. This indicates that the distribution of doctors became unbalanced in Japan between 2006 and 2011.

We apply the cluster analysis technique to the gap data. The following three kinds of data sets are inputs to the cluster analysis: i) bed gap data (by area, by population), ii) doctor gap data (by area, by population), and iii) Bed and Doctor aggregated gap data. The computational results of the cluster analysis are given in Table 5. From Table 5, we find that HKD (Hokkaido) is always exceptional as it constitutes a cluster by itself. This is due to the fact that HKD's allocation of beds and doctors by area is exceptionally far smaller than that in other prefectures as it has an extremely large area. We also find that the Doctor only and the Bed-Doctor aggregate cases give the same clustering result, while the Bed only case gives a slightly different result for clusters II and III. Clusters II and III contain the most populated urban prefectures such as TKY, OSK, FKO, $\mathrm{CHB}, \mathrm{KNG}$, and AIC for all cases. Cluster II for the Doctor and the Bed-Doctor aggregate cases consists of TKY, OSK, and FKO only, which means that all these prefectures have large positive gaps both by area and by population. Cluster III for these cases consists of SIT, CHB, KNG, and AIC, which correspond to the fact that these prefectures have large positive gaps by area and large negative gaps by population.
Table 5 Results of cluster analysis (20II)

\begin{tabular}{ll}
\hline Cluster & Prefectures \\
\hline Beds & HKD \\
II & SIT, CHB, TKY, KNG, AIC \\
III & KGM, ISK, KYT, OSK, OKY, HRS, YGC, TKS, \\
& MIZ, KGS, OKN \\
IV & AOM, IWT, MYG, AKT, YGT, FKS, IBR, TCG, \\
& GNM, NGT, FKI, YMN, NGN, GIF, SZO, MIE, SIG, \\
Doctors & HYG, NAR, WKY, TTR, SMN \\
I & HKD \\
II & TKY, OSK, FKO \\
III & SIT, CHB, KNG, AIC \\
IV & AOM, IWT, MYG, AKT, YGT, FKS, IBR, TCG, \\
& GNM, NGT, TYM, ISK, FKI, YMN, NGN, GIF, \\
& SZO, MIE, SIG, KYT, HYG, NAR, WKY, TTR, \\
& SMN, OKY, HRS, YGC, TKS, KGW, EHM, KOC, \\
& SAG, NGS, KMM, OIT, MIZ, KGS, OKN \\
Beds and Doctors & \\
I I & HKD \\
II & TKY, OSK, FKO \\
III & SIT, CHB, KNG, AIC \\
IV & AOM, IWT, MYG, AKT, YGT, FKS, IBR, TCG, \\
& GNM, NGT, TYM, ISK, FKI, YMN, NGN, GIF, \\
& SZO, MIE, SIG, KYT, HYG, NAR, WKY, TTR, \\
& SMN, OKY, HRS, YGC, TKS, KGW, EHM, KOC, \\
SAG, NGS, KMM, OIT, MIZ, KGS, OKN \\
\hline
\end{tabular}

Abbreviations: HKD, Hokkaido; AOM, Aomori; IWT, Iwate; MYG, Miyagi; AKT, Akita; YGT, Yamagata; FKS, Fukushima; IBR, Ibaraki; TCG, Tochigi; GNM, Gumma; SIT, Saitama; CHB, Chiba; TKY, Tokyo; KNG, Kanagawa; NGT, Niigata; TYM, Toyama; ISK, Ishikawa; FKI, Fukui; YMN, Yamanashi; NGN, Nagano; GIF, Gifu; SZO, Shizuoka; AIC, Aichi; MIE, Mie; SIG, Shiga; KYT, Kyoto; OSK, Osaka; HYG, Hyogo; NAR, Nara; WKY, Wakayama; TTR, Tottori; SMN, Shimane; OKY, Okayama; HRS, Hiroshima; YGC, Yamaguchi; TKS, Tokushima; KGW, Kagawa; EHM, Ehime; KOC, Kochi; FKO, Fukuoka; SAG, Saga; NGS, Nagasaki; KMM, Kumamoto; OIT, Oita; MIZ, Miyazaki; KGS, Kagoshima; OKN, Okinawa.

\section{$\mathrm{LPH}$ reform and its effects $\mathrm{LPH}$ reform}

The chronic financial deficits of LPHs were a long-standing problem until the LPH system was reformed in 2007. The substantial nature of LPHs made the reform politically difficult. Just before the new policy for local governments requiring them to publish financial indicators in 2008 based on accounting data with LPEs was proposed, the MIC launched the Local Public Hospital Reform as a measure to spur local governments to improve the efficiency of the LPHs. Due to the large amount of deficits of LPHs, some local governments faced financial failure and also might have required their being under the direct control by MIC. Financial incentives were introduced to encourage local governments to be fully engaged in the reform. The government introduced subsidies as well as gave permission to local governments to issue bonds to restructure the LPHs and develop a network of other public 
health care facilities. Besides improving the financial condition, improving the efficiency and accountability of LPHs and developing an efficient local health care delivery system were set as the other major objectives.

At the end of 2007, A Planning Guideline for Reforming Local Public Hospitals was published by MIC. Local governments were required to develop a 5-year performance improvement plan in FY2008 according to their practice including the following: 1) performance indicators, such as the occupancy rate, the ratio of ordinary revenue to expenditure, and the ratio of remuneration to expenditure, should be published and used to monitor the performance of LPHs; 2) the health care delivery system should be reorganized by concentrating beds in well-functioning "magnet hospitals" and building "satellite clinics" based on these magnet hospitals in order to secure health care services in rural areas, ie, "reorganizing and networking" should be executed; 3) the management of LPHs should be reviewed, and the organizational reform of LPHs, including outsourcing daily management by introducing the designated manager system and the corporatization or privatization of LPHs, should be initiated on the basis of the review. The guidelines also stipulated that LPHs whose occupancy rates were less than $70 \%$ in the last 3 years should cut the number of beds or be replaced by clinics. ${ }^{16}$

Regarding the changes in the number of hospitals and the number of beds for PRHs and LPHs, during the period from 2005 to 2011, the number of PRHs decreased from 7,241 in 2005 to 6,952 in 2011. The number of PRH beds increased from 1,117 thousand in 2005 to 1,122 thousand in 2007 , and then decreased drastically to 1,101 thousand in 2011 . The decrease of PRH beds might be due to the implementation of the Health Care Reform Act in 2006 which aimed at reducing the number of long-term care beds in hospitals. The number of LPHs and LPH beds have constantly decreased from 1,060 hospitals and 251 thousand beds in 2005 to 968 hospitals and 233 thousand beds in 2011, respectively.

In March, 2012, a survey was conducted by MIC in order to evaluate the implementation of the LPH reform. The percentage of LPHs running a surplus increased from $27.8 \%$ in 2007 to $52.2 \%$ in 2010 . Also, we find that the ratio of ordinary revenue to expenditure increased from $95.2 \%$ to $100.1 \%$ in 2010 , the occupancy rate decreased from $77.5 \%$ to $74.8 \%$, and the ratio of remuneration to expenditure decreased from $55.3 \%$ to $53.3 \%$ during the same period. As the financial performance had been greatly improved after the reform, in the following part we mainly investigate the financial performance between LPHs and private hospitals.

The Hospital Operation Monitoring Analysis Survey (HOMAS) provides the opportunity to observe the financial situation of hospitals under different ownership. The HOMAS is conducted by Japan Hospital Federation (JHF) annually, aimed at monitoring the operation of its member hospitals and providing basic information such as profit, cost, and staff salary to improve the overall management system as well as the payment system. There were approximately 590 LPHs and 290 PRHs included in the survey from 2005 to 2011. As shown in Table 6, the financial performance of PRHs was much better than that of LPHs. The percentage of hospitals running a surplus was approximately 10\% among LPHs, while it was $55 \%$ among private hospitals. The yearly percentage of hospitals running a surplus shows a V-shape in the two groups: the percentages in 2008 were the lowest point, which was $6.7 \%$ for LPHs and $45.5 \%$ for private hospitals.

The financial performance was substantially influenced by the fee schedule system. The revision rate of medical services increased in 2008 for the first time after three consecutive decreases of the revision rate since 2002. The improvement of financial performance is probably related to the increased revision rate.

\section{Evaluating the LPH reform}

According to HOMAS, the revenue of a hospital includes the medical revenue, non-medical revenue, and extraordinary revenue, and the expenditure includes the medical expenditure, non-medical expenditure, and extraordinary losses. Figure 2 shows the revenues and expenditures per 100 beds for LPHs and PRHs during the earlier-mentioned period. During the period from 2005 to 2011, the average revenues were always higher than the expenditures for PRHs except in 2008, while the average expenditures have always been larger than revenues for LPHs. The situation for LPHs worsened from 2005 to 2008 as the expenditures expanded during the earlier period, while the revenues decreased slightly. The situation has started

Table 6 Number and composition of surplus and deficit hospitals by ownership

\begin{tabular}{|c|c|c|c|c|c|c|}
\hline \multirow[t]{2}{*}{ Year } & \multicolumn{3}{|l|}{ LPH } & \multicolumn{3}{|l|}{ PRH } \\
\hline & $\begin{array}{l}\text { Total } \\
\text { (N) }\end{array}$ & $\begin{array}{l}\text { S-H, } \\
\text { n (\%) }\end{array}$ & $\begin{array}{l}\text { D-H, } \\
\text { n (\%) }\end{array}$ & $\begin{array}{l}\text { Total } \\
\text { (N) }\end{array}$ & $\begin{array}{l}\text { S-H, } \\
\text { n (\%) }\end{array}$ & $\begin{array}{l}\text { D-H, } \\
\text { n (\%) }\end{array}$ \\
\hline 2005 & 631 & $72(\mathrm{I} I .4)$ & $559(88.6)$ & 317 & $180(56.8)$ & $137(43.2)$ \\
\hline 2006 & 599 & $56(9.3)$ & $543(90.7)$ & 279 & I 47 (52.7) & I 32 (47.3) \\
\hline 2007 & 594 & $44(7.4)$ & $550(92.6)$ & 307 & 161 (52.4) & I 46 (47.6) \\
\hline 2008 & 599 & $40(6.7)$ & 559 (93.3) & 319 & I 45 (45.5) & $174(54.5)$ \\
\hline 2009 & 584 & $50(8.6)$ & 534 (9I.4) & 323 & $178(55.1)$ & 145 (44.9) \\
\hline 2010 & 579 & $87(15.0)$ & $492(85.0)$ & 307 & 193 (62.9) & II4(37.I) \\
\hline 2011 & 553 & 85 (I5.4) & $468(84.6)$ & 188 & $122(64.9)$ & $66(35.1)$ \\
\hline
\end{tabular}

Note: Data from The Report of Hospital Operation Monitoring Analysis Survey. Tokyo: Japan Hospital Federation. Available from: http://www005.upp.so-net.ne.jp/ byo-ren/2/. Accessed October 15, 2013. Japanese. ${ }^{19}$

Abbreviations: D-H, deficit hospital; LPH, local public hospital; PRH, private hospital; S-H, surplus hospital. 


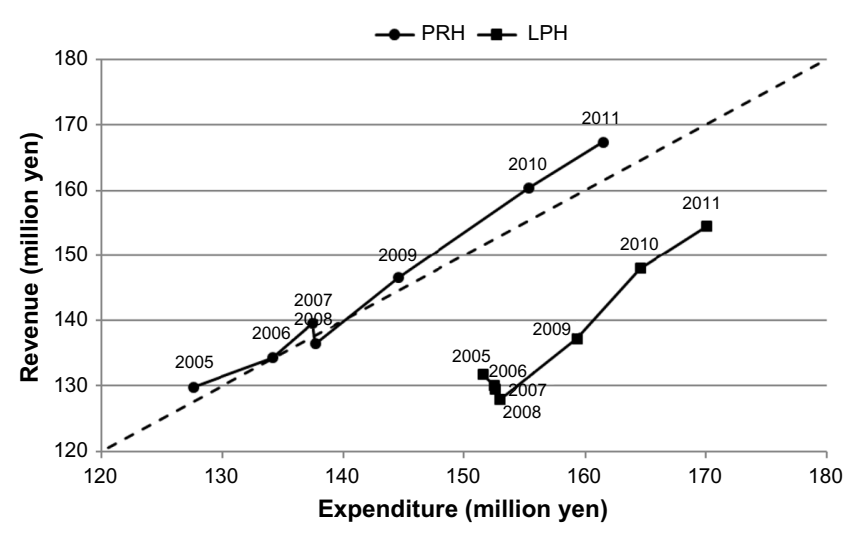

Figure 2 Revenue and expenditure per 100 beds by ownership. Note: Data from The Report of Hospital Operation Monitoring Analysis Survey. Tokyo: Japan Hospital Federation. Available from: http://www005.upp.so-net.ne.jp/ byo-ren/2/. Accessed October 15, 2013. Japanese. ${ }^{19}$

Abbreviations: LPH, local public hospitals; PRH, private hospital.

to improve since 2008 for both LPHs and PRHs. For PRHs, the improvement was mainly attributed to increased revenue, while for LPHs, it was mainly attributed to well-controlled expenditure.

We investigate the differences regarding the revenue and expenditure for PRHs and LPHs by calculating the changes between two consecutive periods. We denote the average revenue and expenditure of LPHs in period $t$ by $L R_{t}$ and $L E_{t}$; respectively, while $P R_{t}$ and $P E_{t}$ stand for the revenue and expenditure for PRHs in period $t$, respectively. Then we define the differences regarding the revenue and expenditure for PRHs and LPHs as follows:

$$
\Delta R_{t}=P R_{t}-L R_{t}, \Delta E_{t}=P E_{t}-L E_{t}
$$

The differences regarding the revenue and expenditure for PRH and LPH are shown in Figure 3. As seen in Figure 3, the

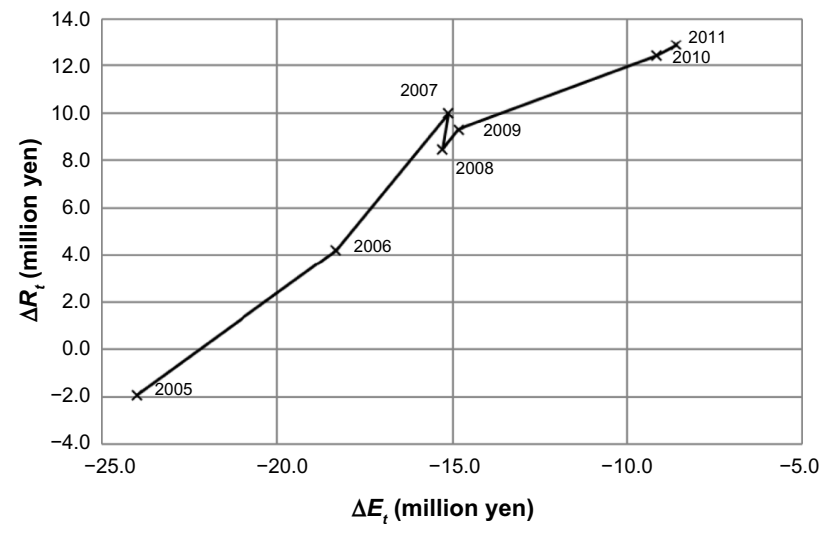

Figure 3 Difference of revenue and expenditure per 100 beds.

Notes: Data from The Report of Hospital Operation Monitoring Analysis Survey. Tokyo: Japan Hospital Federation. Available from: http://www005.upp.so-net.ne.jp/ byo-ren/2/. Accessed October I5, 2013. Japanese. ${ }^{19}$ value of $\Delta R_{t}$ drastically increased between 2005 and 2007, then decreased in 2008. The value of $\Delta R_{t}$ started to increase again after 2008 . While $\Delta E_{t}$ substantially increased among negative values during the period from 2005 to 2007, $\Delta E_{t}$ increased later during the corresponding period from 2008 to 2011 .

We divide the whole period from 2005 to 2011 into two sub-periods: period I (2005-2008) and period II (2008-2011), respectively. During period I, LPHs decreased their revenue by $2.97 \%$, while their expenditure increased by $0.91 \%$. On the other hand, PRHs increased their revenue by approximately $4.83 \%$, while their expenditure increased by $7.34 \%$. Neither expenditure nor revenue changed on a large scale for LPHs during period I; rather, revenue decreased, while expenditure increased slightly. This made the financial situation of LPHs worse. PRHs' large increase in revenue mainly came from inpatient revenue increases. Regarding period II, we find increases in both expenditure and revenue for both LPHs and PRHs. For LPHs, revenue increased $17.2 \%$ during period II, while expenditure increased $10.1 \%$. In the case of PRHs, revenue increased $18.5 \%$ during period II, higher than that for LPHs, while expenditure increased $10.1 \%$, which was also larger than that for LPHs.

Table 7 shows the medical expenditure and revenue for LPHs and PRHs during the period from 2005 and 2011. The medical expenditure of LPHs was more than that of PRHs, but the difference of medical expenditure between PRHs and LPHs decreased annually from 21.0 million yen in 2005 to 6.7 million yen in 2011 , a decrease of more than $68.0 \%$. This major difference came from remuneration. The remuneration of LPHs was 12.0 million yen more than that of PRHs, but it decreased to 4.2 million yen in 2011, or by more than $65.0 \%$. The difference of material expenditure also decreased dramatically during the same period, from 7.0 million yen in 2005 to 0.81 million yen in 2011 , or by $88.5 \%$.

Looking at the ratio of medical expenditure to medical revenue during the period from 2005 to 2011 for LPHs and PRHs in Table 7, we find that the medical expenditure of LPHs was always approximately $15 \%$ higher than the medical revenue, while PRHs could achieve a balance between the two. For LPHs, the ratios firstly increased from $13.3 \%$ higher in 2005 to $18.3 \%$ higher in 2008, then decreased to $9.3 \%$ higher in 2011. For PRHs, the ratios started to decrease from $1.6 \%$ higher in 2008 , to achieving balance in 2008 , and further to expenditures being just $96.2 \%$ of the medical revenue in 2010 .

Figure 4 shows $\Delta E_{t}$ and $\Delta R_{t}$ for each category of both expenditure and revenue for LPHs and PRHs during the period from 2005 to 2011. During period I, $\Delta E_{t}$ decreased, which was mainly due to the decreases of both remuneration 
Table 7 Medical expenditure and medical revenue for PRHs and LPHs

\begin{tabular}{|c|c|c|c|c|c|c|c|}
\hline Financial indicators & 2005 & 2006 & 2007 & 2008 & 2009 & 2010 & 2011 \\
\hline \multicolumn{8}{|c|}{ Private hospital (PRH) } \\
\hline Medical expenditure & $|24,94|$ & 131,612 & 134,330 & 134,745 & $|4|, 704$ & 152,436 & 158,566 \\
\hline Remuneration & 64,494 & 67,902 & 70,201 & 70,855 & 75,503 & 80,498 & 83,778 \\
\hline Material & 29,829 & 31,754 & 31,596 & 30,589 & 32,707 & 36,472 & 38,118 \\
\hline Utility fee & 22,483 & 23,629 & 24,108 & 24,557 & 24,851 & 26,025 & 27,200 \\
\hline Others & 8,136 & 8,326 & 8,425 & 8,744 & 8,642 & 9,442 & 9,470 \\
\hline Medical revenue & 127,080 & 132,083 & 136,756 & 134,089 & 144,008 & 158,005 & 164,784 \\
\hline Inpatient & 85,224 & 88,565 & 92,819 & 91,772 & 98,138 & 108,852 & 112,623 \\
\hline Out-of-pocket & 2,919 & 2,646 & 3,032 & 2,993 & 3,138 & 2,908 & 2,920 \\
\hline Outpatient & 34,826 & 36,231 & 36,327 & 34,682 & 38,194 & 40,642 & 43,691 \\
\hline Others & $4, I I I$ & $4,64 I$ & 4,578 & 4,642 & 4,537 & 5,603 & 4,641 \\
\hline \multicolumn{8}{|c|}{ Local public hospital (LPH) } \\
\hline Medical expenditure & 145,922 & $|46,75|$ & 146,924 & 147,798 & 154,106 & $|59,76|$ & 165,288 \\
\hline Remuneration & 76,540 & 77,309 & 78,515 & 78,797 & 82,579 & 85,947 & 88,014 \\
\hline Material & 36,828 & 36,749 & 35,385 & 34,209 & 36,390 & 37,404 & 38,924 \\
\hline Utility fee & 21,472 & 21,352 & 21,493 & 22,862 & 23,496 & 24,535 & 25,607 \\
\hline Others & 11,082 & $|I, 34|$ & $|1,53|$ & 11,930 & $1 \mathrm{I}, 640$ & $\mathrm{II}, 875$ & $|2,74|$ \\
\hline Medical revenue & 128,775 & 127,362 & 126,722 & 124,949 & $|33,78|$ & 144,877 & 151,192 \\
\hline Inpatient & 85,160 & 84,790 & 84,582 & 84,475 & 90,105 & 98,997 & $103,00 \mid$ \\
\hline Out-of-pocket & 1,270 & $\mathrm{I}, 287$ & 1,335 & $\mathrm{I}, 352$ & $\mathrm{I}, 427$ & $\mathrm{I}, 442$ & $\mathrm{I}, 484$ \\
\hline Outpatient & 40,705 & 39,371 & 38,786 & 37,208 & 40,334 & 42,114 & 44,478 \\
\hline Others & $\mathrm{I}, 64 \mathrm{I}$ & 1,915 & 2,018 & 1,914 & 1,916 & 2,323 & 2,229 \\
\hline
\end{tabular}

Notes: Values represent I thousand Yen. Data from The Report of Hospital Operation Monitoring Analysis Survey. Tokyo: Japan Hospital Federation. Available from: http:// www005.upp.so-net.ne.jp/byo-ren/2/. Accessed October 15, 20I3. Japanese. ${ }^{19}$

and material costs. During period II, remuneration continued to decrease while material costs decreased on a large scale. The values of utility fees and others, which had rather small shares, were essentially stable during the whole period. The decreases of remuneration in $\Delta E_{t}$ resulted from the rapid increase of remuneration in PRHs, while LPHs' remuneration was rather stable during period I and slightly increased in the second period.

Regarding the trend of $\Delta R_{t}$, it decreased drastically during period I, which was mainly due to the difference of inpatient revenues. As shown in Table 7, the medical revenue of PRHs per 100 beds was more than that of LPHs except in 2005. The

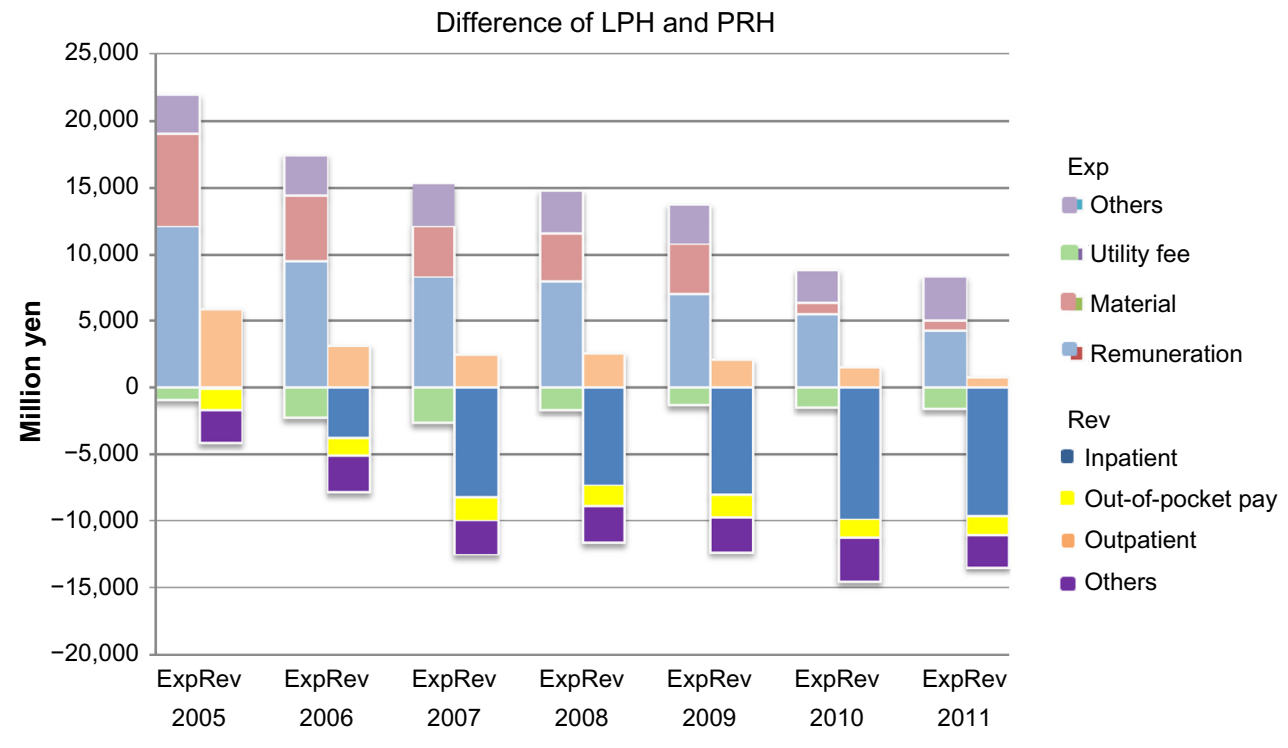

Figure 4 Difference of medical revenue between PRHs and LPHs per 100 beds.

Note: Data from The Report of Hospital Operation Monitoring Analysis Survey. Tokyo: Japan Hospital Federation. Available from: http://www005.upp.so-net.ne.jp/byoren/21. Accessed October 15, 2013. Japanese. ${ }^{19}$

Abbreviations: Exp, expenditure; LPH, local public hospital; PRH, private hospital; Rev, revenue. 
difference decreased from 1.7 million yen in 2005 to -13.6 million yen in 2011. The revenue of inpatient services contributed to the major difference, from less than 1 million yen in 2005 to 9.6 million yen in 2011. The large increase of inpatient revenue in the first period resulted from PRHs' large revenue increase, and that in the second period was due to large revenue increases in both periods I and II. Even though inpatient revenue did not show a large increase during period II, it was rather stable at approximately 10 million yen for both PRHs and LPHs during the corresponding period. For $\Delta R_{t}$, the values of outpatient were slightly increasing during the whole period, while the remaining component, others, was almost constant. The outpatient revenue of LPHs was more than that of PRHs; however, the difference of outpatient revenue decreased from 5.9 million yen in 2005 to 0.8 million yen in 2011 .

Although there are several shortcomings in comparing the annual data between the two groups, such a comparison could help in understanding the different impacts of the health policies on PRHs and LPHs. HOMAS was not a follow-up type survey, and the subjects were different from year to year. Under the context of the supply side fee control system, the financial performance may have been substantially influenced by the fee schedule. The revision rate of medical services increased in 2008 for the first time after three consecutive decreases of the revision rate since 2002. The improvement of the financial performance of LPHs and PRHs after 2008 probably resulted from this revision of the fee schedule. However, when we investigate the details of the ratio of medical expenditure to medical revenue between LPHs and PRHs, we find that PRHs seemed to be more capable of increasing revenue while LPHs focused more on expenditure control. The difference was, we believe, probably because of the Local Public Hospital Reform, which was conducted in late 2007.

\section{Summary and conclusion}

The health care system of Japan is characterized by its universal coverage health insurance system. Due to the pressure of increasing health expenditures, the Japanese government had been exerting great efforts to control excessive hospital resources. However, the increasing medical care needs due to the aging population have made the problem of insufficient medical staff more serious. We can say that the Japanese government has been facing the dilemma of attaining both the reduction in the number of medical care facilities and the concurrent increase in the medical labor force, as shown in our paper where we find an increase in the number of hospital staff with a shrinking number of hospitals and beds. In this paper, we briefly described the health care delivery system in Japan. Then we investigated the distribution of hospital resources and evaluated the impacts of the LPH reform from several different viewpoints such as the equality of local hospital resources and the financial condition of LPHs.

Accessibility is determined by the medical care delivery system. Under the private sector-dominated medical care delivery system in Japan, fair accessibility may face potential challenges; namely, PRHs are more likely to pursue profit rather than public functions. Large increases in inpatient revenue in the first period (2005-2008) resulted from PRHs' large revenue increases, and those in the second period (2008-2011) were due to large revenue increases for both PRHs and LPHs during periods I and II. In addition, our findings reveal that hospital resources are concentrated in the nine major developed and populated prefectures: $\mathrm{HKD}$, TKY, OSK, FKO, SIT, CHB, KNG, AIC, and HYG. Further analysis indicates that the density of hospital resources is higher in underdeveloped prefectures in the southern part of Japan.

The LPH system is considered as an important measure to guarantee fair accessibility, which has played a crucial role in securing medical services for local residents. Our analysis finds that prefectures in the northern parts of Japan, which had a larger proportion of LPH beds, faced scarce hospital resources. Their counterparts in the south, however, were ranked at the bottom in terms of the proportion of LPH beds and at the top in terms of the density of hospital resources. Clarifying the difference among these underdeveloped prefectures, which may result from multiple factors, requires a more careful scrutiny of LPH-related policies to prevent compromising the equity of the medical care delivery system.

We also evaluated the impacts of the LPH reform launched in 2007. The data from HOMAS revealed that after the LPH reform, private and LPH adopted different strategies to enhance their financial soundness. The PRHs focused on increasing revenue, while LPHs controlled expenditure.

The gap analysis indicates that although the total number of physicians increased throughout the research period, the physician gap among prefectures also increased. More physicians were absorbed by developed prefectures. We believe downsizing or even closing LPHs because of local financial pressure aggravated the unfair distribution of physicians, although the medical staff training policy and other related health policies may also play a major role at the same time. ${ }^{14,17}$ 
In this paper, we only took into account the population and territorial area when we measured the equality of hospital resources. Age, health status, and many other socioeconomic factors which are important to justify these inequalities are not included in this paper. Second, we only investigated the financial performance of LPHs. The change of efficiency or productivity, which is more important to evaluate a reform, is not included in this research study. Further, the LPH reform has been criticized for improving LPH financial accounts at the expense of increasing the national burden, ${ }^{18}$ but our paper does not address this critical question. We suggest that further research on the efficiency of LPHs and the impacts on local medical expenditure be conducted in the future.

\section{Disclosure}

The authors report no conflicts of interest in this work.

\section{References}

1. Jeong H-S, Hurst J. An assessment of the performance of the Japanese health care system. Labour Market and Social Policy Occasional Papers. 2001; No 56, OECD Publishing.

2. Hashimoto H, Ikegami N, Shibuya K, et al. Cost containment and quality of care in Japan: is there a trade-off? Lancet. 2011;378(9797): 1174-1182.

3. Jones RS. Health-care reform in Japan: controlling costs, improving quality and ensuring equity. OECD Economics Department Working Papers. 2009; No 739, OECD Publishing.

4. Hamada H, Lapalme-Remis S. International perspective on mixed health care: Japan. McGill J Med. 2008;11(1):79-80.

5. Ikegami N, Yoo BK, Hashimoto H, et al. Japanese universal health coverage: evolution, achievements, and challenges. Lancet. 2011; 378(9796):1106-1115.

6. Ikegami N, Campbell JC. Japan's health care system: containing costs and attempting reform. Health Aff. 2004;23(3):26-36.
7. Annual Health, Labour and Welfare Report 2012-2013 [database on the Internet]. Ministry of Health, Labour and Welfare. 2013 [accessed on July 10, 2015 ]. Available from: http://www.mhlw.go.jp/english/wp/ wp-hw7/index.html.

8. Survey of Medical Institutions. Ministry of Health, Labour and Welfare; 2002-2013. Available from: http://www.mhlw.go.jp/toukei/list/79-1a. html. Accessed April 15, 2015.

9. OECD Health Statistics. Paris: OECD Publishing; 2011. Available from: http://www.oecd-ilibrary.org/social-issues-migration-health/data/ oecd-health-statistics_health-data-en. Accessed July 11, 2015.

10. MIC. 2007. Chiho Koritsu Byouin Kaikaku Gaidorain [A Guideline for Reforming Local Public Hospitals]. Tokyo: Ministry of Internal Affairs and Communications. Japanese. Available from: http://www.soumu. go.jp/main_sosiki/c-zaisei/hospital/pdf/071224_zenbun.pdf. Accessed March 15, 2013.

11. Tanaka H. Administrative Reform in Japanese Local Governments. Papers on the Local Governance System and its Implementation in Selected Fields in Japan. 2010; No 18. Tokyo: Council of Local Authorities for International Relations; 2010.

12. Nomura $\mathrm{K}$, Inoue $\mathrm{S}$, Yano E. The shortage of pediatrician workforce in rural areas of Japan. Tohoku J Exp Med. 2009;217(4):299-305.

13. Matsumoto M, Inoue K, Bowman R, Kajii E. Self-employment, specialty choice, and geographical distribution of physicians in Japan: a comparison with the United States. Health Pol. 2010;96(3):239-244.

14. Toyabe $\mathrm{S}$. Trend in geographic distribution of physicians in Japan. Int J Equity Health. 2009;8(5).

15. Shinjo D, Aramaki T. Geographic distribution of healthcare resources, healthcare service provision, and patient flow in Japan: a cross sectional study. Soc Sc Med. 2012;75(11):1954-1963.

16. Matsuda R. Reform of local public hospitals. Health Policy Monitor. 2008.

17. Teo A. The current state of medical education in Japan: a system under reform. Med Educ. 2007;41(3):302-308.

18. Kawaguchi H, Tone K, Tsutsui M. Estimation of the efficiency of Japanese hospitals using a dynamic and network data envelopment analysis model. Health Care Manag Sci. 2014;17(2):101-112.

19. The Report of Hospital Operation Monitoring Analysis Survey. Tokyo: Japan Hospital Federation. Available from: http://www005.upp.so-net. ne.jp/byo-ren/2/. Accessed October 15, 2013. Japanese.

\section{Publish your work in this journal}

Risk Management and Healthcare Policy is an international, peerreviewed, open access journal focusing on all aspects of public health, policy, and preventative measures to promote good health and improve morbidity and mortality in the population. The journal welcomes submitted papers covering original research, basic science, clinical \& epidemio-

\section{Dovepress}

logical studies, reviews and evaluations, guidelines, expert opinion and commentary, case reports and extended reports. The manuscript management system is completely online and includes a very quick and fair peerreview system, which is all easy to use. Visit http://www.dovepress.com/ testimonials.php to read real quotes from published authors. 